\title{
Compatibility of the concept of adaptation level with crossover interactions caused by distribution biases
}

\author{
YASUHARU OKAMOTO ${ }^{1}$ \\ Department of Behavioral Science, Faculty of Letlers, Kanazawa University, Kanazawa, Ishikawa 920
}

\begin{abstract}
Birnbaum (1974) 's crossover interaction effect caused by variations of stimulus distributions was cited by Parducci (1983) as an incompatible evidence with the adaptation-level theory (Helson, 1964). In this report, the author showed that a non-log-linear version of the adaptation-level theory can predict the crossover interaction, in contrast with Parducci. There remains a possibility that the stimulus frequency affected on sensory processes.
\end{abstract}

Key words: adaptation-level theory, range-frequency model, psychological judgment, stimulus distribution.

According to Parducci (1983), his rangefrequency model is formulated as follows;

1) $C_{i}=b \cdot J_{i}+a$

2) $J_{i}=w \cdot R_{i}+(1-w) \cdot F_{i}$

3) $R_{i}=\left(S_{i}-s_{\mathrm{min}}\right) /\left(s_{\mathrm{max}}-s_{\mathrm{m} \mid \mathrm{n}}\right)$

4) $F_{i}=\left(r_{i}-1\right) /(N-1)$

where $C_{i}$ is the overt category rating of the presented stimulus $i, J_{i}$ the judgment, $a$ and $b$ constants which match the endpoints of the judgment scale with those of the category scale, $R_{i}$ the range value, $F_{i}$ the frequency value, $w$ the weight parameter, $s_{i}, s_{\min }$ and $s_{\max }$ the stimulus values of the presented, lowest and highest stimuli, and $r_{i}$ the rank of the stimulus $i$ within the $\mathcal{N}$ stimuli. One of the characteristics of the range-frequency model is that psychological impression or sensation of the given stimulus is not affected by the exposure to the immediately preceding one. On the contrary, the adaptation-level(AL) theory (Helson, 1964) assumes that responses are determined in relation to the current $\mathrm{AL}$, which is the pooled effect of three classes of stimuli, focal, background (or contextual) and residual ones. That is, sensation is affected by AL. AL is not necessarily

1 The programs were written in TURBO PAS$\mathrm{CAL}$ (Borland International Inc.) and run on PC8801 system with CP/M (NEC). The figures were plotted by DXY-980 (Roland DG Corp.) controlled by PC8801 system. constant during experimentation. It fluctuates trial by trial, depending on the presented stimulus (self-adaptation). When we consider the effect of this self-adaptation on the next trial, we should incorporate into the model the local contextual effect of the immediately preceding trial on the present one. On the other hand, the range-frequency model neglects this local effect, because both the range value $R_{i}$ and the frequency value $F_{i}$ are determined based on the whole stimulus set.

In criticizing AL theory, Parducci (1983) and Birnbaum (1974) contended that AL theory cannot explain the crossover interactions shown in Figs. 3 and 5 in Birnbaum (1974) (here, Figs. 1 and 2), while the range-frequency model does well. Anderson (1982) also cited Birnbaum (1974)'s crossover interaction as one of evidences that sensation to the particular stimulus is constant, although he admits the possibility of nonconstancy. In this report, the author showed that these crossover interactions can be explained by the model based on the concept of adaptation level.

Birnbaum (1974) outlined the psychophysical judgment as the composition of two functions; (a) the psychophysical function,

$$
\phi=H(\phi)
$$

relating the psychological impression $(\phi)$ 
to the physical measure of the stimulus $(\phi)$ (here, this process is abbreviated as sensation): (b) the response function,

$$
R=J\left(\varphi^{\prime}\right)
$$

relating responses $(R)$ to the impressions (this process is abbreviated as judgment). Given the stimulus set, the range-frequency model assumes that sensation $H$ is constant $R_{i}$ for the particular stimulus. The crossover interactions are interpreted as the results of the effects on judgment of stimulus distributions. These interactions seem to reject the possibility of the contextual effects on sensations. However, this incompatibility comes from the special form, the log-linear one, of AL theory. Adoption of other nonlinear form of the effect of adaptation level makes it possible to predict the crossover interactions, which means that the sensation of the particular stimulus is affected by the context.

\section{Non Log-Linear Version of AL Theory}

If the immediately preceding stimulus $s_{k}$ affects the current $\mathrm{AL}$, the sensation for the focal stimulus $s_{i}$ should be represented as $H_{\mathrm{AL}_{k}}\left(s_{i}\right)$, where $\mathrm{AL}_{k}$ represents the current $\mathrm{AL}$ affected by the immediately preceding stimulus $s_{k}$. With the assumption that the response function $J$ is the identity one for simplicity, the average rating $R_{u \text { r }}$ for the stimulus $s_{i}$ is calculated as

$$
R_{\mathrm{ave}}\left(s_{i}\right)=(1 / \mathcal{N}) \sum_{k=1}^{N} H_{\mathrm{AL}_{k}}\left(s_{i}\right)
$$

where $N$ is the total number of stimuli.

$$
W=0.90
$$

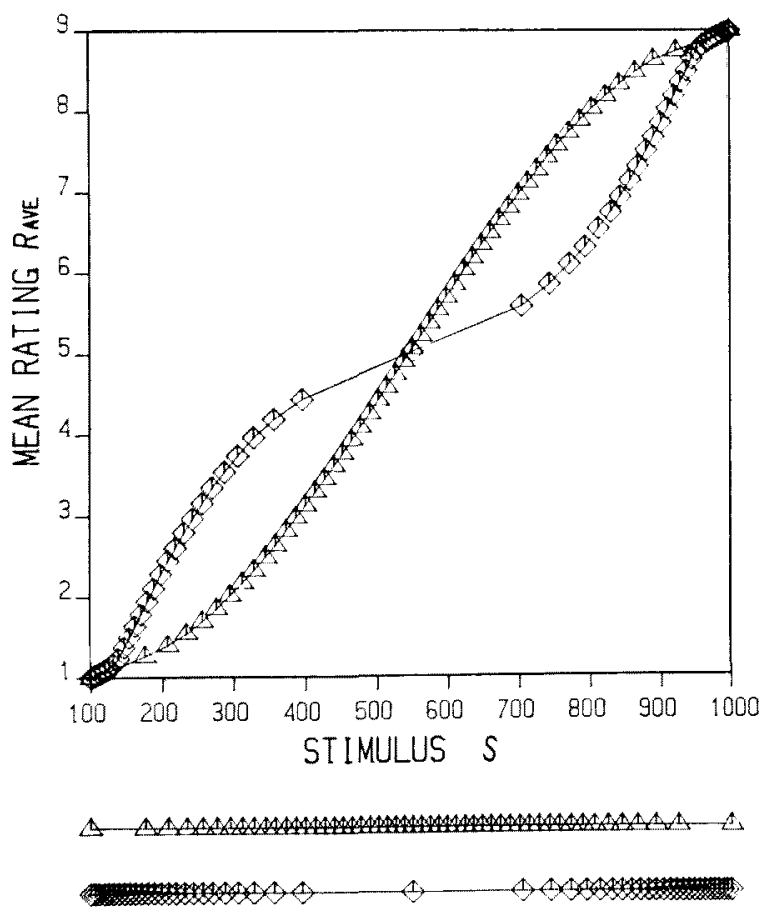

Fig. 1. Predicted average ratings for the quadratic distribution of stimuli, which are also displayed under the graph. 
Differentiating eq (1) w.r.t. s, we get

$$
\frac{d}{d s} R_{\text {uve }}(s)=(1 / \mathcal{N}) \sum_{k=1}^{N} \frac{d}{d s} H_{A L_{k}}(s)
$$

If $H_{\mathrm{AL}_{k}}(s)$ contains the variable $A \mathrm{~L}_{k}$ in a $\log$-linear form, i.e.

$$
H_{\mathrm{AL}_{k}}(s)=f(s)+a \cdot \log \left(\mathrm{AL}_{k}\right),
$$

the curves $H_{\mathrm{AL}_{k}}(s)$ for different $k$ are parallel to each other, because

$$
\frac{d}{d s} H_{\mathrm{AL}_{\mathrm{k}}}(s)=f^{\prime}(s) \text {, }
$$

which does not contain $\mathrm{AL}_{k}$. In this case, $R_{a \nabla r} s$ for different stimulus distributions do not cross each other.

But, eq (3) should be regarded as one of possible approximations to $H_{\mathrm{AI}_{\mathrm{k}}}(s)$. AL is defined as the zero of function (Helson,
1964). But, why does the organism adjust his zero-level of sensation depending on the surrounding environment? Klinke (1981) writes that adaptation enhances discrimination of sound pressure levels. Helson (1964) also noted dependency of discriminability on AL. If shift of AL causes change in discriminability,

$$
\frac{d}{d s} H_{\mathrm{AL} \cdot \boldsymbol{k}}(s)
$$

must contain the variable $\mathrm{AL}_{k}$. In this report, as an example, $H_{\mathrm{AI}_{*}}(s)$ is specified as follows;

$$
\begin{aligned}
& H_{\mathrm{AL}_{k}}(s) \\
& \quad=\left\{\begin{array}{ll}
\log \left(1+s-\mathrm{AL}_{k}\right), & \text { for } s \geqq \mathrm{AL}_{k} \\
-\log \left(1+\mathrm{AL}_{k}-s\right), & \text { for } s<\mathrm{AL}_{k}
\end{array}\right. \text { (4) }
\end{aligned}
$$

The slope of eq(4) is steepest at $\mathrm{AL}_{k}$,

$$
W=0.90
$$
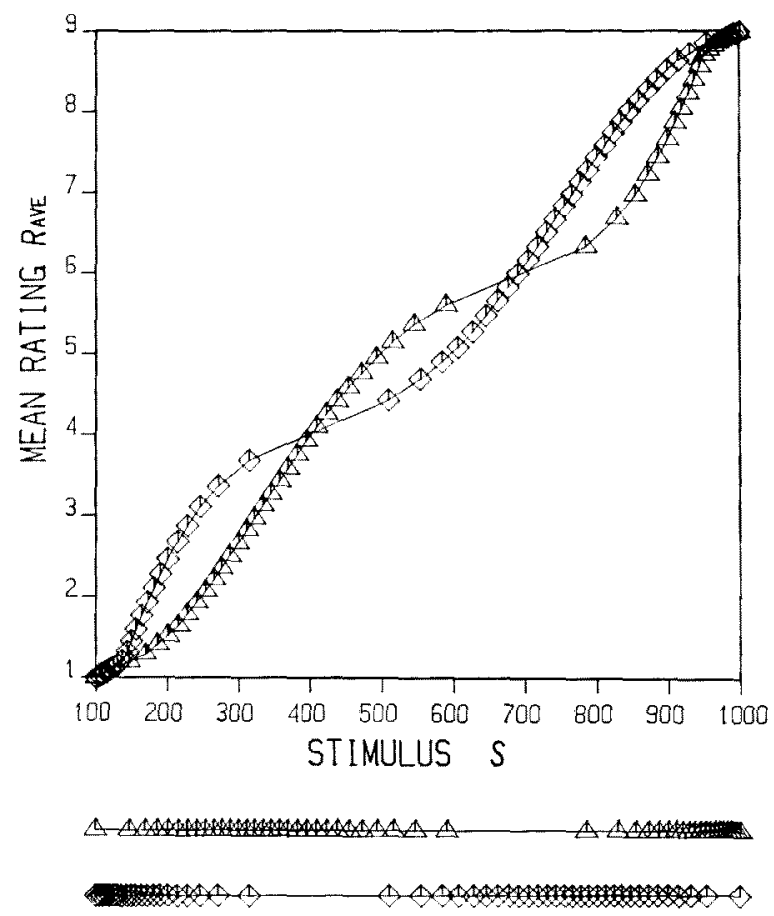

Fig. 2. Predicted average ratings for the cubic distributions of stimuli, which are also displayed under the graph. 
so shift of $\mathrm{AL}_{\mathrm{k}}$ causes other than vertical shift of $H_{\mathrm{AL}_{k}}(s)$, which explains the crossover interactions.

In calculating values $R_{\mathrm{are}}\left(s_{i}\right)$ for the biased distributions, $A L_{k}$ is specilied as

$$
\mathrm{AL}_{k}=w \cdot s_{k}+(1-w) \cdot s_{\mathrm{uve}},
$$

where $s_{k}$ and $s_{\text {ave }}$ are the immediately preceding stimulus value and the base line value of $\mathrm{AL}$ which is here set to be an arbitrarily chosen value near the midpoint in the given stimulus range, and $w$ is a weight parameter.

$R_{\mathrm{av}}\left(s_{i}\right) \mathrm{s}$ are plotted in Figs. 1 and 2 for quadratic and cubic distributions of stimuli with parameter values $w=0.9$ and $s_{\mathrm{ave}}=500$. In these figures, $R_{\mathrm{aven}} s$ are linearly transformed so that the ratings to the maximum and minimum stimulus values, 100 and 1000 , are 1 and 9 . The crossover interactions shown in these figures are essentially the same as those in Birnbaum (1974), i.e. the non-loglinear $\mathrm{AL}$ model is also consistant with the crossover interactions.

For the purpose of qualitative analysis, $H_{\mathrm{AL}}(s)$ was defined rather arbitrarily as eq(4), which has characteristic of the steepest gradient at $\mathrm{AL}$. Precise specification of $H_{\mathrm{AL}}(s)$ requires experimental data, which will determine $J$ and $H$ from the same data as in Birnbaum (1974), or independently from separate data. But, Birnbaum (1974) attributed the effects of distribution biases to the frequency values $F_{i}$, and fitted the context-free psychophysical function to the residuals. We could take the other extreme position, i.e. at- tribute the contextual effects to psychophysical lunctions and obtain a contextfree judgment function $J$ from the residuals. In this procedure, effects of distribution biases would be attributed to effects on sensation. If the truth is between the two extremes, we should not determine the both effects on sensation $H$ and judgment $J$ simultaneously. Better way is, e.g., to determine $H$ and $J$ from separate data. But, whatever form $H_{\mathrm{AL}}(s)$ takes as the precise one, it should be noted here that any function $H_{\mathrm{AL}}(s)$ with the steepest gradient about at $\mathrm{AL}$ makes $R_{\mathrm{av} \theta}(s)$ be steeper in dense regions of stimulus distributions than in scarce ones, i.e., the crossover interactions in Birnbaum (1974), because eq $(2)$ shows that the gradient of $R_{\mathrm{ave}}(s)$ is the average of those of $H_{\mathrm{AL}}(s)$.

\section{References}

Anderson, N. H. 1982 Methods of information integration theory. Now York: Academic Press.

Birnbaum, M.H. 1974 Using contextual effects to derive psychophysical scales. Perception and Psychophysics, 15, 89-96.

Helson, H. 1964 Adaptation-level theory. New York: Harper and Row, Publishers.

Klinke, R. 1981 Physiology of hearing. In R. F. Schmidt (Ed.), Fundamentals of sensory physiology. New York, Heidelberg, Berlin: Springer-Verlag. Pp. 180-204.

Parducci, A, 1983 Category ratings and the relational character of judgment. In H. Geissler (Ed.), Modern issues in perception. Amsterdam, New York, Oxford: North-Holland. Pp. 262282.

(Received April 18, 1985; accepted Jan. 18, 1986) 